\title{
Modeling and simulation of forces applied to the horizontal axis wind turbine rotors by the vortex method coupled with the method of the blade element
}

\author{
Ibtissem Barkat ${ }^{1}$, Abdelouahab Benretem², Fawaz Massouh ${ }^{3}$, Issam Meghlaoui ${ }^{4}$, Ahlem Chebel ${ }^{5}$ \\ 1,2,4,5 Laboratory, Electromechanical Engineering, Badji-Mokhtar-Annaba University, Annaba, Algeria \\ ${ }^{3}$ Laboratory DynFluid, Arts et Métiers ParisTech, 151 The Boulevard Hospital, Paris, France
}

\begin{tabular}{l} 
Article Info \\
\hline Article history: \\
Received Apr 26, 2020 \\
Revised Jan 14, 2021 \\
Accepted Feb 4, 2021 \\
\hline
\end{tabular}

Keywords:

Forces on the blade Method of the blade element Modeling

Vortex method

Wind power

\begin{abstract}
This article aims to study the forces applied to the rotors of horizontal axis wind turbines. The aerodynamics of a turbine are controlled by the flow around the rotor, or estimate of air charges on the rotor blades under various operating conditions and their relation to the structural dynamics of the rotor are critical for design. One of the major challenges in wind turbine aerodynamics is to predict the forces on the blade as various methods, including blade element moment theory (BEM), the approach that is naturally adapted to the simulation of the aerodynamics of wind turbines and the dynamic and models (CFD) that describes with fidelity the flow around the rotor. In our article we proposed a modeling method and a simulation of the forces applied to the horizontal axis wind rotors turbines using the application of the blade elements method to model the rotor and the vortex method of free wake modeling in order to develop a rotor model, which can be used to study wind farms. This model is intended to speed up the calculation, guaranteeing a good representation of the aerodynamic loads exerted by the wind.
\end{abstract}

This is an open access article under the CC BY-SA license.

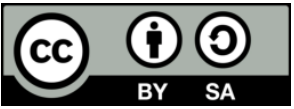

\section{Corresponding Author:}

Barkat Ibtissem

Laboratory: Electromechanical Engineering

Badji-Mokhtar-Annaba University, 23000, Algeria

Email: bessouma2007@ hotmail.com

\section{INTRODUCTION}

Use of wind energy as a source of renewable energy was considerably expanded in the 20th century, with the growing penetration of wind energy generation into power grids [1], [2]. Lanchester was the primary predicter of a perfect wind's greatest power [3]. One of the faremost vital challenges within the aerodynamics of wind turbines is to predict precisely the forces on the blade, which are modeled by various approaches with varying degrees of complexity and precision, including the method of the blade element the vortex model and approaching the Navier-Stokes equations using CFD. Today, a BEM-based engineering model is commonly used to analyze the aerodynamic performance of a wind turbine where it relies on a normal and uniform flow hypothesis and on aerodynamic charges acting on an actuating drive rather than on a finite number of blades [4]. Hansen, et al., [5] show that the accuracy of the BEM under normal load conditions is reasonable if sufficient aerodynamic profile data is given. The method of the blade element is defined as the improved model of the Rankine-Froude dynamics theory [6], [7], and that was the firs model to predict the influx rotor speed where it is assumed that the rotor can be replaced with uniformly charged disk. CFD method is to resolve the differential equations of fluid mechanics. Navier-Stokes equations or simplification as the Euler equations, potential, potential linearized and RANS, by algorithms and numerical methods [8]. 
Comprehensive review is presented in Sanderse, et al., [9] on the use of CFD codes for wind turbine calculations. The analytical techniques are easily defined according to the turbulence modeling method used, which is of particular significance for the modelling of wind turbine wakes. The vortex methods are another approach, which is more reliable than the theory of momentum, and less expensive than CFD. Methods based on vorticity have various formulations, from basic analytical models to more complex numerical methods [10]. The vortex theory can also be used to estimate the aerodynamic efficiency of wind turbines, based on the potential flow, non-viscous. The advantage of the method is that it reduces the complexity of the flow and it has generally been used for airfield and aircraft aerodynamic analysis. Despite not being able to predict the viscous phenomena such as separation and boundary layer, drag, its combination with aerodynamic profile data actually tabulated a powerful method for predicting the fluid flow. Methods of free vortex wake have developed as robust, flexible tools for modeling the aerodynamic loads [11]. Yu, et al., [12] developed a free wake method that uses a vortex ring model with a semi-infinite cylindrical vortex tube where near wake is simplified in to a series of vortex rings and the far wake with a semi-infinite cylindrical vortex tubes. Branlard and Gaunaa [13] developed approach based on the use of vortex-based methods including cylindrical and helical wake models to study the unstable effects of yaw, shear and turbulent flows. Another work of Vaal [14] presented an aerodynamic model that combined Kutta-Joukowski theory with the blade element model, which predict the blade charges accurately and effectively using the time-evolution of the induced speeds. Recent work by Jing Dong, et al., [15] suggested a modified method of free-wake vortex rings to resolve the aerodynamic load around wind turbines with a horizontal axis, on fixed and floating support structures.

In this work, we propose an aerodynamic model which allows to quickly simulate the flow of a wind turbine with horizontal axis. This model makes it possible to precisely determine and calculate the position of the vortices and the induced radial and axial speeds at any point in the wake. A free wake vortex method has been implemented coupled with the blade elements method which allows us to quickly determine the aerodynamic loads.

\section{PRESENTATION OF THE MODEL}

During operation of a wind, the helical wake is formed behind a rotor, the latter which is modeled with a series of vortex rings [16]-[18]. For the modeling of the wind turbine rotor, one can use the theory of blade element which consists the blade divided into several parts.

\subsection{Modeling of the wake}

The wake is modeled by a series of vortex rings Figure 1, ready to move axially and to widen radially, able to interact with the impact of the speed field induced in the wake. Increased passage of the blade refers to the separation of the intensity of the vortex ring and the radius $\mathrm{R}$, which is proportional to the axial force applied to the rotor.

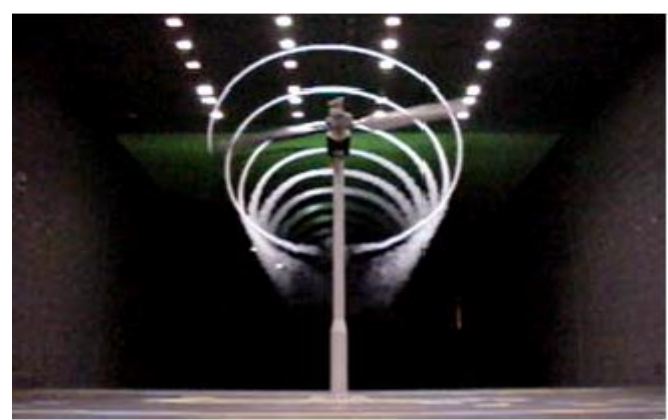

(a)

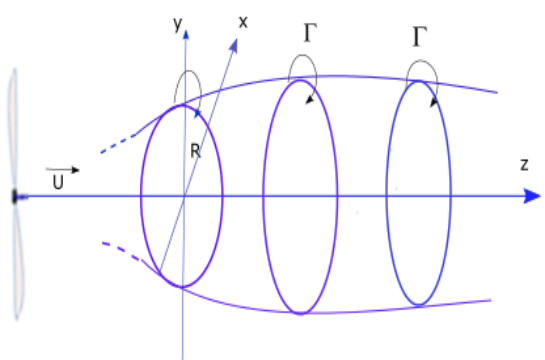

(b)

Figure 1. (a) Wind wake visualization [19], (b) representation of the helical track by vortex rings

\subsection{Velocity induced by a vortex ring}

The velocity ild induced with the vortex rings is determineted using the law of Biot-Savart. Figure 2 shows geometry of vortex rings with a radius $\mathrm{R}$, located in plane $\mathrm{xy}$. The induced velocity is calculated as (1): 


$$
d v_{i}=\frac{\Gamma}{4 \pi} \frac{r}{|r|^{3}}
$$

$\Gamma$ is the intensity of the vortex segment.

Modeling the vortex rings by law Biot-Savart involves singularities within the vortex cores, so the obtained solution is, singular if the analysis point $\mathrm{P}$ falls on the ring, to avoid singular behavior, a regularization parameter $\delta$ is introduced. Figure 3 depicts the axial velocity induced with and without the regularisation parameter.

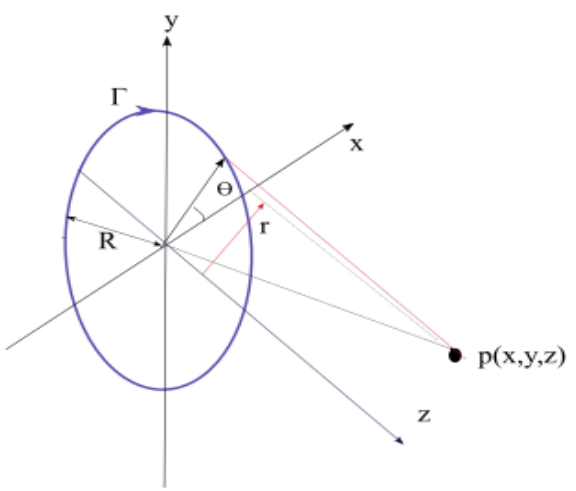

Figure 2. Geometry of the vortex ring in the plane xy

$$
d v_{i}=\frac{\Gamma \times r}{4 \pi\left(|r|^{2}+\delta^{2}\right)^{3 / 2}}
$$

Additionally, the precise resolution is voiced with the elliptical integrals of first and second kind at any point in the flux field [20] Thus, the radial and axial parts of the induced velocity are expressed in the following (3) (4) $\mathrm{V}_{\mathrm{z}} \mathrm{Vr}$ for the ring vortex with the radius $\mathrm{R}$ and also the intensity $\Gamma$.

$$
\begin{aligned}
& v_{r}(r, z)=\frac{-r . z}{2 \pi \sqrt{z^{2}+(R+r)^{2}+\delta^{2}}} \times\left[K(s)-\frac{R^{2}+r^{2}+z^{2}+\delta^{2}}{z^{2}+(R-r)^{2}+\delta^{2}} E(s)\right] \\
& v_{z}(r, z)=\frac{-r}{2 \pi \sqrt{z^{2}+(R+r)^{2}+\delta^{2}}} \times\left[K(s)-\frac{R^{2}+r^{2}+z^{2}+\delta^{2}}{z^{2}+(R-r)^{2}+\delta^{2}} E(s)\right]
\end{aligned}
$$

Where the elliptic integrals $\mathrm{K}(\mathrm{s})$ and $\mathrm{E}(\mathrm{s})$ complete first and second kind in parameter $\mathrm{S}(5)$ :

$$
S=\frac{4 r R}{Z+(R-r)^{2}+\delta^{2}}
$$

The axial location of the vortex ring in the free wake is calculated with the addition of a wind speed $U_{0}$, the self-induced velocity and mutually induced velocities of control point, as suggested by Heemst, et al., (6) [21].

$$
v_{a}=u_{0}+\sum_{i=1}^{j} v_{z i}+v_{\text {aut }}
$$

Self-induced velocity is defined at the point situated in the vortex ring for radius $\mathrm{R}$ and radius core $r_{c}$, according to the (7), as suggested by Lewis [22]. 


$$
v_{\text {auti }}=\frac{\Gamma}{4 \pi R}\left(\log \frac{8 R}{r_{c}}-\frac{1}{4}\right)
$$

The radial velocity of the vortex ring is determined in a control point to the periphery of the vortex ring by adding all mutually induced velocities (8).

$$
v_{r}=\sum_{i=1}^{j} v_{r i} v_{a u t i}
$$

From Figure 4 We see that the vortex rings, which are formed right behind the rotor, are located in an area of high radial velocity, which causes their diameter to increase rapidly when away from the rotor. Such radial velocities, measured by the control point at the edge of the vortex ring, are reduced to zero. Figure 5 demonstrate the axial velocity as a function of the distance $\mathrm{z}$ of the rotor plane on the edge of the rings. It is remarkable that the vortex ring behind the rotor travels initially more slowly than those located further away from the rotor plane.

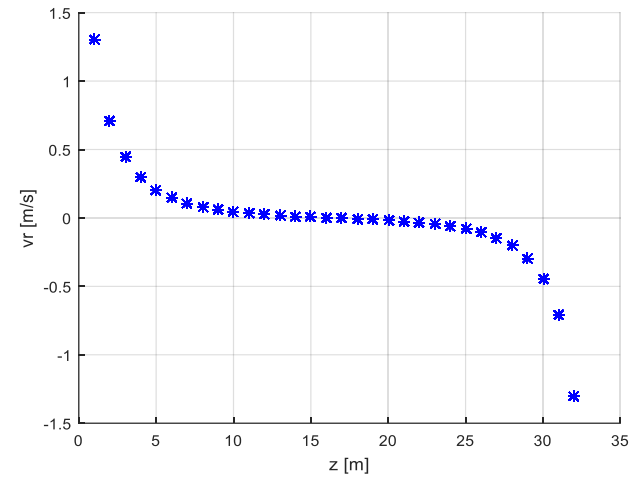

Figure 4. Distribution of radial velocity of the vortex ring along the wake

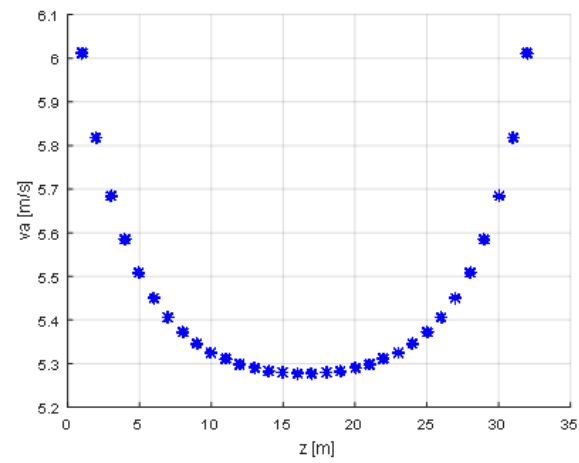

Figure 5. Distribution of axial velocity along the wake

\subsection{Modeling of the blades}

The aerodynamic modeling of the blade is of great importance in the design of the wind rotors, because very detailed calculations have to be carried out in order to determine the different essential dimensions of the blade. Use the blade element method to determine the force applied to the rotor blade, Figure 6 illustrate the speed triangle of blade element. In this approach, the blade is divided into segments of $\mathrm{k}$, or each is assumed to be independent of the others. For each section the induced velocity is evaluated axially and tangentially by the vortex system, as shown by Karpatne, et al., [23].

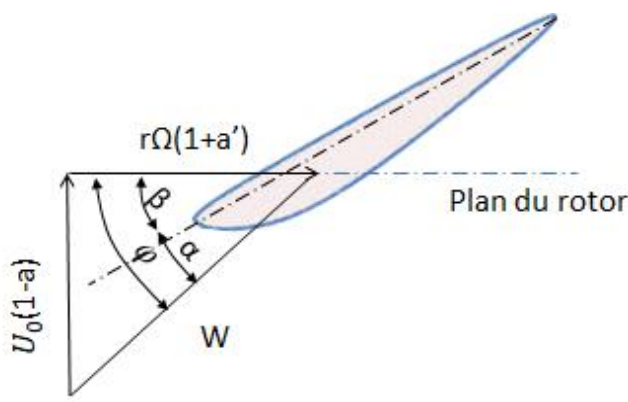

Figure 6. Speed triangle of blade element 
The angle of incidence is given by (9) and (10):

$$
\begin{aligned}
& \alpha=\varphi-\beta \\
& \varphi=\tan ^{-1}\left(\frac{u_{0}(1-a)}{r \Omega\left(1+a^{\prime}\right)}\right)
\end{aligned}
$$

The coefficient of local lift cl (i) is determinated for each $\alpha$ i after consulting the data table of the airfoil of the blade. The total thrust $\mathrm{T}$ generated by the rotor is given by (11):

$$
T=\frac{1}{2 k} \sum_{i}^{k} N \rho w^{2} c l(i) c(i) \Delta r
$$

In order to calculate the intensity for each blade, the Kutta-Joukowski theorem is used. The equivalente vortex intensity can be determined from the total turbine thrust (12) [24]

$$
T=\Gamma \rho \int_{0}^{R} \Omega r d r
$$

By assuming that all the resulting flow is dragged from the tip of the blades and adding the total thrust of all the blades, the intensity of the vortex ring is determinated by (13) [24].

$$
\Gamma=\frac{2 T}{\rho N R^{2} \Omega}
$$

Where $\Gamma$ is the circulation that will be released from the vortex ring. This iterative method for $\Gamma$ is performed at each time step $\Delta t$, as shown in Karpatne, et al., (14) [23].

$$
\Delta t=\frac{2 T}{N \Omega}
$$

\subsection{Scheme of temporal integration}

At each time step, the total field of velocity induced by all the rings shall be calculated. For the passing of time a predictor-corrector scheme has been selected as shown in (15) and (16) [25]. As shown in (15) and (16) are used for the first iteration to predict a solution of axial and radial positions of the vortex rings.

$$
\begin{aligned}
& r_{i}^{*}=r_{i}^{n}+\Delta t v_{i r}\left(r_{i}^{n}, z_{i}^{n}\right) \\
& z_{i}^{*}=z_{n}^{i}+\Delta t v_{i z}\left(r_{i}^{n}, z_{i}^{n}\right)
\end{aligned}
$$

The Adams-Bashforth method is used for precision and stability reasons to determine the location of the vortex rings. As shown in (17) to (18).

$$
\begin{aligned}
& r_{i}^{*}=r_{i}^{n}+\frac{3}{2} \Delta t v_{i r}\left(r_{i}^{n}, z_{i}^{n}\right)-\frac{1}{2} \Delta t V_{i r}\left(r_{i}^{n-1}, z_{i}^{n-1}\right) \\
& z_{i}^{*}=z_{n}^{i}+\frac{3}{2} \Delta t v_{i z}\left(r_{i}^{n}, z_{i}^{n}\right)-\frac{1}{2} \Delta V_{i z}\left(r_{i}^{n-1}, z_{i}^{n-1}\right)
\end{aligned}
$$

Thereafter, the speeds $\left(\left(v_{i r}\left(r_{i}^{*}, z_{i}^{*}\right), v_{i z}\left(r_{i}^{*} z_{i}^{*}\right)\right)\right.$ are determinated in this new position). The ring location is determined by average intermediate velocity and new velocies (19) and (20).

$$
r_{i}^{n+1}=r_{i}^{n}+\frac{\Delta t}{2}\left[v_{i r}\left(r_{i}^{*}, z_{i}^{*}\right)+v_{i r}\left(r_{i}^{*}, z_{i}^{*}\right)\right]
$$




$$
z_{i}^{n+1}=z_{i}^{n}+\frac{\Delta t}{2}\left[v_{i z}\left(r_{i}^{*}, z_{i}^{*}\right)+v_{i z}\left(r_{i}^{*}, z_{i}^{*}\right)\right]
$$

\section{RESULTS AND DISCUSSION}

In this work, we have used two types of wind turbines to study and simulate the forces applied to wind turbine rotors. The first case is a wind-rotor type NREL5MW at wind speed of $8 \mathrm{~m} / \mathrm{s}$. The NREL report [26] contains some basic parameters for turbines, aerodynamic profiles, aerodynamic characteristics and other turbine details used in this model. Figure 7 shows the distribution of vortex rings in the wake, the rings move axially and expand radially.

This numerical simulation us allows the following results presented in Figures 9 and 10 which show the distribution of the intensity $\Gamma$, the tangential and the axial forces distribution along the blade. In Figure 8 it is noted that the intensity $\Gamma$ blade tip is $39 \mathrm{~m}^{2} / \mathrm{s}$ this intensity increases proportionally to the value of 0.87 $\mathrm{r} / \mathrm{R}$ and decreases rapidly in the blade root.

The tangential and the axial forces distribution in the rotor plane is sown in Figure 9 (a) (b). Note that these forces increases slightly at the end of the blade and stabilizes, after these forces decreases rapidly at the blade roots. The second case is a wind turbine: Gamesa G58 with an NACA 4412 profile 3 blades, the rotor at a radius of 58 meters from the results obtained in Figure 10 shows the power recovered by the turbine Gamesa according to the wind speed.

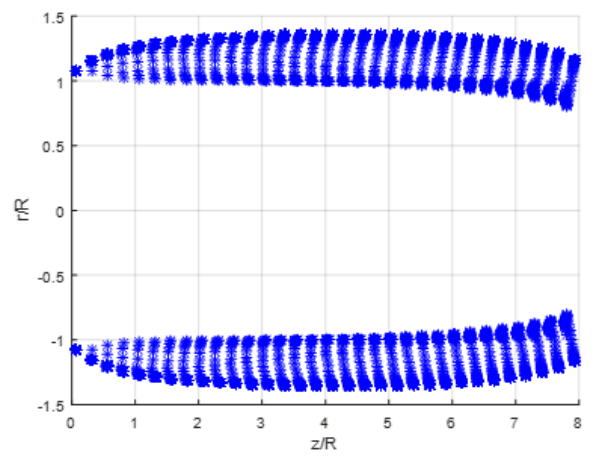

Figure 7. Vortex rings distrbution in the wake

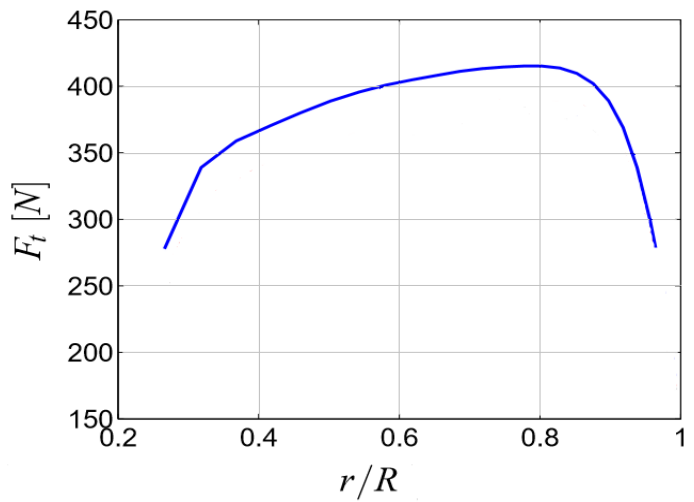

(a)

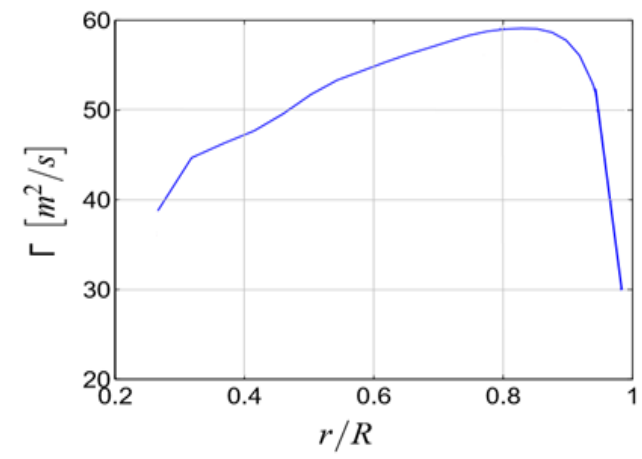

Figure 8. Distribution of intensity $\Gamma$ along the blade

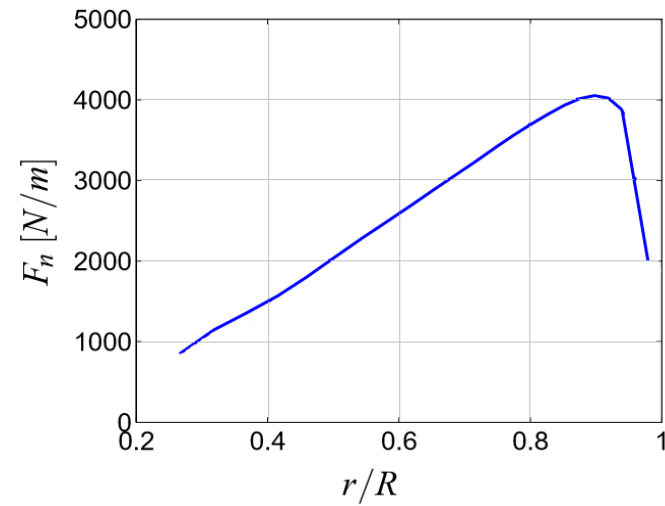

(b)

Figure 9. (a) The tangential forces distribution in the rotor plane, (b) the axial forces distribution in the rotor plane 


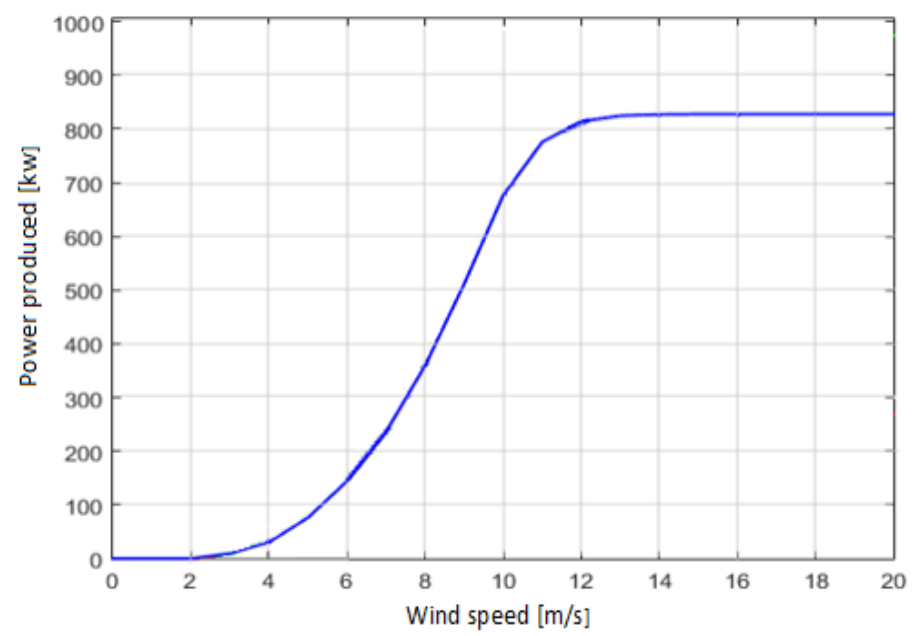

Figure 10. Power produced by gamesa G58 according to the wind speedy

\section{CONCLUSION}

In this paper, we presented a work that involves the modeling study of the forces applied to the wind turbine rotor axis horizontal. To do this, we have proposed a model that combines the method of blade element for determination of aerodynamic forces and vortex theory to calculate the velocity field induced in the rotor plane. This model has allowed the implementation of a model digital capable of performing simulations with acceptable accuracy and a time adequate calculation. Numerical simulations are performed and the simulation results of the two cases of wind turbines show the validation of this model to determine the wind turbine performance and various aerodynamic parameters blades. It is possible to consider the proposed model as operational and can also be used to simulate unstable cases.

\section{REFERENCES}

[1] "Energy outlook 2016," BP p.l.c, BP Statistical, 2016.

[2] Hachemi, Glaoui, Harrouz, Abdelkader, Ismail, Messaoudi, Hamid, Saab, "Modeling of Wind Energy on Isolated Area," International Journal of Power Electronics and Drive System (IJPEDS), vol. 4, no. 2, pp. 274-280, 2014.

[3] Lanchester, F. W., "A contribution to the theory of propulsion and the screw propeller," Journal of the American Society for Naval Engineers, vol. 27, no. 2, pp 57-98, 1915.

[4] Hamidreza, Abedi, "Development of Vortex Filament Method for Aerodynamic Loads on Rotor Blades," Thesis for licentiate of engineering, no. 22, 2013.

[5] Hansen, M., Sørensen, J., Voutsinas. S., Sørensen. N., Madsen, H., "State of the art in wind turbine aerodynamics and aeroelasticity," Progress in Aerospace Sciences, vol. 42, no. 4, pp. 285-330, 2006.

[6] W. J. M. Rankine, "On the Mechanical Principle of the Action of Propellers," Transactions of the Institute of Naval Architects, vol. 6, 1865.

[7] W. Froude, "On the Elementary Relation between Pitch, Slip and Propulsive Efficiency," Transactions of the Institute of Naval Architects, vol. 19, 1978.

[8] Naji Abdullah Mezaal, Osintsev K. V, Alyukov S.V., "The computational fluid dynamics performance analysis of horizontal axis wind turbine," International Journal of Power Electronics and Drive System (IJPEDS), vol. 10, no. 2, pp. 1072-1080, 2019

[9] B. Sanderse, S. P. Van der Pijl, and B. Koren, "Review of computational fluid dynamics for wind turbine wake aerodynamics," Wind Energy, vol. 14, no. 7, 2011.

[10] Emmanuel, B., "Wind Turbine Aerodynamics and Vorticity-Based Methods, Fundamentals and Recent Applications," Springer, Germany, 2017.

[11] Bofeng, Xu, Tongguang, Wang, Yue, Yuan, Zhenzhou, Zhao and Haoming, Liu, "A Simplified Free Vortex Wake Model of Wind Turbines for Axial Steady Conditions," Applied Sciences, vol. 8, no. 6, p. 866, 2018

[12] Yu. W, Ferreira. C.S, van. Kuik, G. Baldacchino, D., "Verifying the blade element momentum method in unsteady, radially varied, axisymmetric loading using a vortex ring model," Wind Energy, vol. 20, no. 2, pp. 269-288, 2017.

[13] Branlard. E, S. P, \& Gaunaa. M., "Analysis of wind turbine aerodynamics and aeroelasticity using vortex based methods," DTU Wind Energy, mo. 0052, 2015.

[14] DeVaal. J., "Aerodynamic Modelling of Floating Wind Turbines," Ph.D.Thesis, NTNU Trondheim, Norway, 2015.

[15] Jing. Dong, Axelle, Viré, Carlos Simao, Ferreira, Zhangrui, Li and Gerard van Bussel, "A Modified Free Wake Vortex Ring Method for Horizontal-Axis Wind Turbines," Energies, vol. 12, no. 20, p. 3900, 2019.

[16] J. B. de Vaal, M. O. L. Hansen; and T. Moan, "Validation of a vortex ring wake model suited for aeroelastic simulations of floating wind turbines," Journal of Physics: Conference Series, vol. 555, no. 1, pp. 1-10, 2014.

Modeling and simulation of forces applied to the horizontal axis wind turbine rotors by ... (Ibtissem Barkat) 
[17] E. Branlard, M. Gaunaa, "Cylindrical vortex wake model: right cylinder," Wind Energy, vol. 18, no. 11, pp. 973987, 2014.

[18] Ian S. Sullivan, Joseph J., Niemel, A., Robert E., Hershberger, Diogo Bolster and Russel J., Ddonnelly, "Dynamics of thin vortex rings," Journal of Fluid Mechanics, vol. 609, no. 25, pp. 319-347, 2008.

[19] Jean-Jacques. Chattot, "Wind turbine aerodynamics, analysis and design," International Journal of Aerodynamics, vol. 1, no. 3-4, pp. 404-444, 2011.

[20] Chattot, Jean-Jacques, "Actuator Disk Theory-Steady and Unsteady Models," Journal of Solar Energy Engineering vol. 136, no. 3, pp. 031012-031022, 2014.

[21] Van. Heemst, J. W., et al., "Coupling of a free wake vortex ring near-wake model with the Jensen and Larsen farwake deficit models," Journal of Physics: Conference Series, vol. 625, no. 1, 2015

[22] Lewis, Reginald Ivan, "Vortex element methods for fluid dynamic analysis of engineering systems," Cambridge University Press, 2009.

[23] Karpatne. Anand, et al., "Vortex Ring Model of Tip Vortex Aperiodicity in a Hovering Helicopter Rotor," Journal of Fluids Engineering, vol. 136, no. 7, pp. 071104. 2014.

[24] Issam. Maghlaoui, Ivan, Dobrev, Fawas, Massouh, Ouahab, Benretem, Dalila, Khalfa, "Computationally Inexpensive free vortex method to obtain vortex core position in the wake of a horizontal axis wind turbine," International Journal of Fluid Mechanics Research, vol. 44, no. 5, pp. 427-443, 2017.

[25] V. L. Okulov, et al., "The rotor theories by Professor Joukowsky: Vortex theories," Progress in Aerospace Sciences, vol. 73, pp. 19-46, 2015.

[26] J. Jonkman, S. Butterfield, W. Musial, G. Scott, "Wind Turbine for Offshore System Development, 5-MW Reference,” Tech. Rep. TP-500-3806, NREL, 2009. 